\title{
Surgical resection of an intravascular superior vena cava primary lipoma
}

\author{
Pierre Mordant, MD, Olaf Mercier, MD, Elie Fadel, MD, PhD, Ashok Muniappan, MD, \\ Dominique Fabre, MD, Olivier Chataigner, MD, Sacha Mussot, MD, and Philippe G. Dartevelle, MD, \\ Le Plessis Robinson, France
}

Primary tumors of the major central veins are rare. Although benign leiomyomatous tumors are well described, only 4 cases of intravascular lipoma of the superior vena cava (SVC) have been reported. ${ }^{1-4}$ We present here the radiologic findings of 1 such case and discuss why surgical treatment is mandated for this benign and often asymptomatic disease.

\section{CLINICAL SUMMARY}

A 55-year-old woman was referred to our surgical department for enlargement of the superior mediastinum found on a routine chest radiograph. Her medical history and physical examination were unremarkable. Thoracic computed tomography (CT) with intravenous contrast showed an intraluminal nonenhancing tumor occluding the distal right subclavian vein, the right brachiocephalic vein, and the SVC up to the right atrium (Figure 1, $A$ and $B$ ). A superior

\footnotetext{
From the Department of Thoracic and Vascular Surgery and Heart-Lung Transplantation, Marie-Lannelongue Hospital, Le Plessis Robinson, France.

Received for publication March 15, 2009; accepted for publication April 11, 2009. Address for reprints: Olaf Mercier, MD, Department of Thoracic and Vascular Surgery and Heart-Lung Transplantation, 133, Avenue de la Resistance, 92350 Le Plessis Robinson, France (E-mail: o.mercier@ccml.fr).

J Thorac Cardiovasc Surg 2010;140:1437-8

$0022-5223 / \$ 36.00$

Copyright (C) 2010 by The American Association for Thoracic Surgery doi:10.1016/j.jtcvs.2009.04.072
}

vena cavogram revealed a total occlusion of the right subclavian and brachiocephalic veins and of the SVC to the level of the azygos vein. Abnormal collaterals arising from the right axillary and jugular veins reperfused the partially occluded distal SVC through the azygos network (Figure 1,C). Magnetic resonance imaging was consistent with a fatty intravascular lesion. There was no fludeoxyglucose F 18 uptake on positron emission tomographic scan.

The surgical approach combined a median sternotomy with a right transclavicular cervicotomy, allowing control of the SVC as well as the right subclavian, right internal jugular, and azygos veins. A transverse venotomy was made in the SVC just cephalad to the azygos vein to control the distal edge of the tumor. A lobulated fatty tumor was found to be occupying the lumen of the SVC; however, the tumor was free of adhesions to the SVC wall. The origin of the tumor was the junction of the right subclavian and internal jugular veins and was adherent to the wall of the junction between the right innominate vein and SVC as well (Figure 2, A). An en bloc resection was performed that encompassed the entire tumor and its attachments (Figure 2, $B)$. An end-to-end anastomosis was performed between the left innominate vein and the SVC (Figure 2,C). On gross anatomic inspection, the tumor measured 9 by $5 \mathrm{~cm}$. Histologic findings were consistent with a benign intravascular lipoma; they also confirmed a complete resection. The patient's postoperative course was uneventful. After 1 year

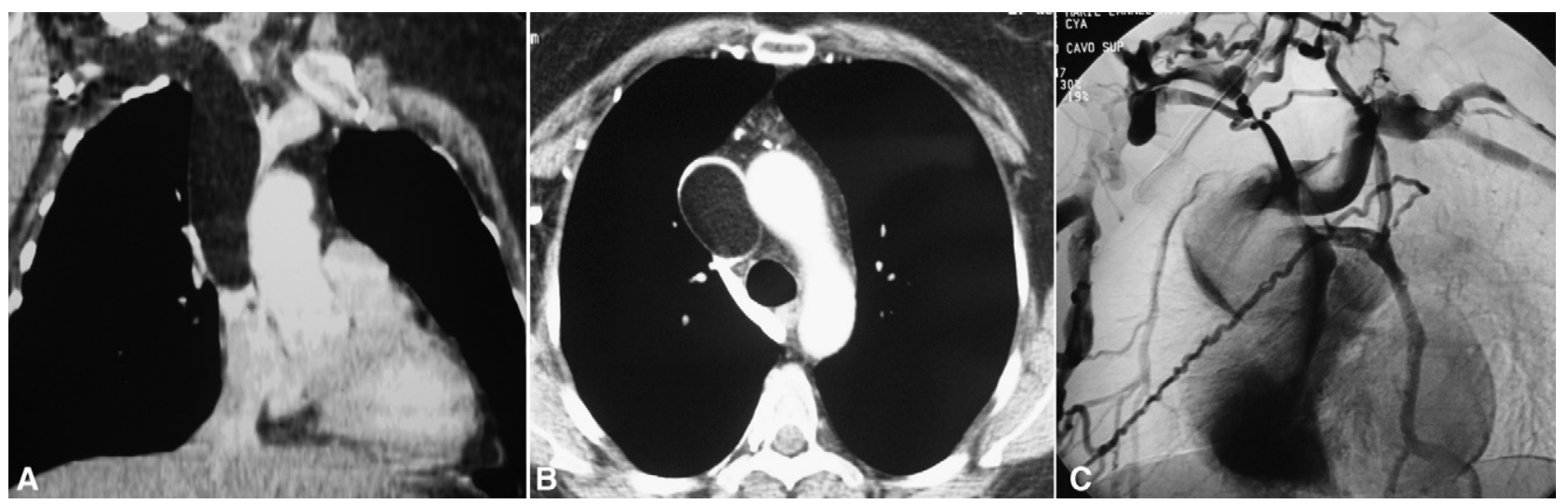

FIGURE 1. Preoperative intravenous contrast-enhanced thoracic computed tomographic scan showing the hypodense tumor filling the lumen of the superior vena cava down to the cavoatrial junction in coronal section (A) and axial section (B). C, Preoperative cavography showing the filling defect of the superior vena cava with venous collaterals. 


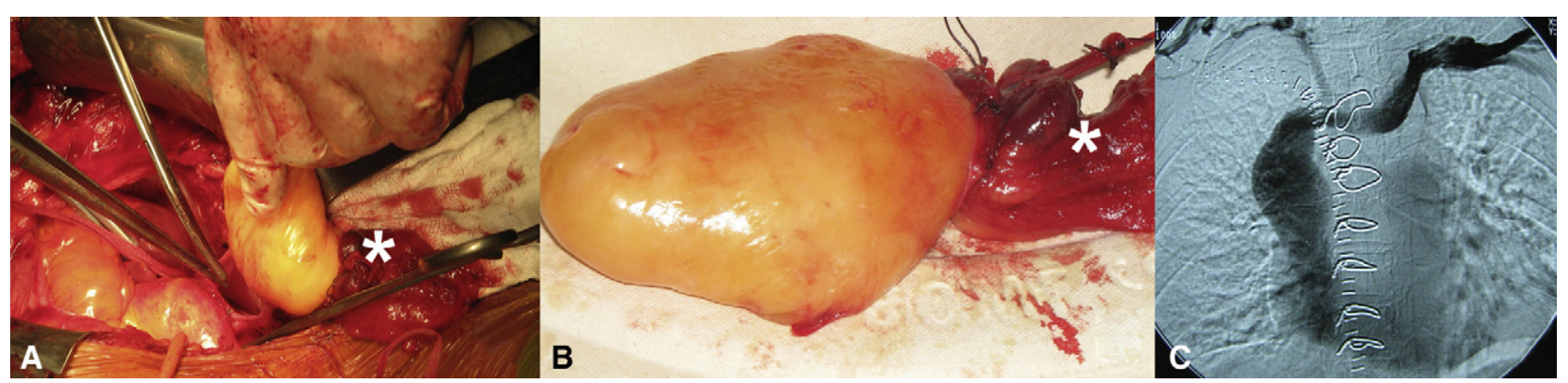

FIGURE 2. A, Operative view showing the extraction of the tumor from the lumen of the superior vena cava. White star marks the tumor implantation at the level of the right subclavian-internal jugular vein junction. B, Gross anatomy of the lipomatous tumor. C, Postoperative cavography showing the anastomosis between the left innominate vein and the unobstructed superior vena cava.

of follow-up, the patient is doing well, without evidence of recurrence on CT scan.

\section{DISCUSSION}

Intravascular lipomas are rare benign tumors that arise from the wall of veins, most commonly within the inferior vena cava. They may be identified in as many as $0.5 \%$ of all CT scans. ${ }^{5}$

Intravascular lipomas may be asymptomatic, or they may induce venous obstructive symptoms, including upper limb swelling. In most cases, the lipoma is incidentally revealed by imaging. In our case, the lipoma was an incidental finding after an abnormal plain chest radiograph. The patient was free of symptoms, despite evidence of occlusive disease identified on venography. The diagnosis of a benign intravascular lipoma is arrived at by CT scan, magnetic resonance imaging, superior vena cavogram, and fludeoxyglucose $\mathrm{F} 18$ positron emission tomographic scan. Contrast-enhanced CT reveals a well-defined rounded mass of fat attenuation occluding the lumen of the SVC. Magnetic resonance imaging confirms the fatty nature of the mass and its intraluminal localization. Superior vena cavography typically shows a reduced lumen of the SVC and a large lobulated filling defect. In our case, abnormal venous collaterals on the superior vena cavogram testified to the compromised venous return and the risk of SVC thrombosis. Fludeoxyglucose F 18 PET scan results are typically negative, ruling out the diagnosis of venous extension of a distinct primary cancer.

Of the 4 cases previously described in the literature, 2 cases did not lead to surgical resection because the mass was not symptomatic, ${ }^{1,4}$ and 1 patient underwent only partial resection. ${ }^{2}$ In contradistinction, we believe that surgical resection is mandatory for 3 reasons. First, it is impossible to make a firm distinction between a benign lipoma and a liposarcoma before surgical resection. Second, the growth of the tumor may eventually induce symptomatic SVC obstruction or enter the right atrium. Finally, the surgical treatment is safe and requires neither prosthetic bypass nor lifelong anticoagulation.

It is our conviction that surgery for large and benign intravascular tumors must be safe and complete. We therefore chose a transsternal and transclavicular approach to control all the major thoracic veins. This technique avoids pulmonary embolism, minimizes bleeding, and allows en bloc resection of the tumor and its area of implantation. The described combination of surgical approaches and anesthetic techniques permits a complete surgical resection of intravascular lipoma of the SVC that establishes the pathologic diagnosis and prevents the complications that may arise from this tumor.

\section{References}

1. Thorogood SV, Maskell GF. Case report: intravascular lipoma of the superior vena cava-CT and MRI appearances. Br J Radiol. 1996;69:963-4.

2. Vinnicombe S, Wilson AG, Morgan R, Saunders K. Intravascular lipoma of the superior vena cava: CT features. J Comput Assist Tomogr. 1994;18:824-7.

3. Trabut JB, Duong Van Huyen JP, Artru B, Bruneval P. [Intravascular lipoma of the superior vena cava]. Ann Pathol. 1999;19:529-31. French.

4. Moore FO, Petersen SR, Norwood SH. Intravascular lipoma of the right innominate vein in a trauma patient. J Am Coll Surg. 2008;207:139.

5. Perry JN, Williams MP, Dubbins PA, Farrow R. Lipomata of the inferior vena cava: a normal variant? Clin Radiol. 1994;49:341-2. 